\title{
ON HODGE THEORY OF SINGULAR PLANE CURVES
}

\author{
NANCY ABDALLAH
}

\begin{abstract}
The dimensions of the graded quotients of the cohomology of a plane curve complement $U=\mathbb{P}^{2} \backslash C$ with respect to the Hodge filtration are described in terms of simple geometrical invariants. The case of curves with ordinary singularities is discussed in detail. We also give a precise numerical estimate for the difference between the Hodge filtration and the pole order filtration on $H^{2}(U, \mathbb{C})$.
\end{abstract}

\section{INTRODUCTION}

The Hodge theory of the complement of projective hypersurfaces have received a lot of attention, see for instance Griffiths [10] in the smooth case, Dimca-Saito [5] and Sernesi [12] in the singular case. In this paper we consider the case of plane curves and continue the study initiated by Dimca-Sticlaru [7] in the nodal case and the author [1] in the case of plane curves with ordinary singularities of multiplicity up to 3.

In the second section we compute the Hodge-Deligne polynomial of a plane curve $C$, the irreducible case in Proposition 2.1 and the reducible case in Proposition 2.2. Using this we determine the Hodge-Deligne polynomial of $U=\mathbb{P}^{2} \backslash C$ and then we deduce in Theorem 2.7 the dimensions of the graded quotients of $H^{2}(U)$ with respect to the Hodge filtration.

In section three we consider the case of arrangements of curves having ordinary singularities and intersecting transversely at smooth points and obtain a formula in Theorem 3.1 generalizing the formulas obtained in [7] and in [1] (for this type of curves). In fact, the results in [1] show that this formula holds in the more general case of plane curves with ordinary singularities of multiplicity up to 3 (without assuming transverse intersection).

In the forth section we show that the case of plane curves with ordinary singularities of multiplicity up to 4 (without assuming transverse intersection) is definitely more complicated and the formula in Theorem 3.1 has to be replaced by the formula in Theorem 4.1 containing a correction term coming from triple points on one component through which another component of $C$ passes.

In the final section we give some applications, we hope of general interest, expressing the difference between the Hodge filtration and the pole order filtration on $H^{2}(U, \mathbb{C})$ in terms of numerical invariants easy to compute in given situations, see Theorem 5.1 and its corollaries. One example involving a free divisor concludes this note.

2000 Mathematics Subject Classification. Primary 32S35, 32S22; Secondary 14H50.

Key words and phrases. plane curves, Hodge and Pole order filtrations . 


\section{Hodge Theory of Plane Curve complements}

For the general theory of mixed Hodge structures we refer to [2] and [14]. Recall the definition of the Hodge-Deligne polynomial of a quasi-projective complex variety $X$

$$
P(X)(u, v)=\sum_{p, q} E^{p, q}(X) u^{p} v^{q}
$$

where $E^{p, q}(X)=\sum_{s}(-1)^{s} h^{p, q}\left(H_{c}^{s}(X)\right)$, with $h^{p, q}\left(H_{c}^{s}(X)\right)=\operatorname{dim} G r_{F}^{p} G r_{p+q}^{W} H_{c}^{s}(X, \mathbb{C})$, the mixed Hodge numbers of $H_{c}^{s}(X)$.

This polynomial is additive with respect to constructible partitions, i.e. $P(X)=$ $P(X \backslash Y)+P(Y)$ for a closed subvariety $Y$ of $X$. In this section we determine $P(C)$ for a (reduced) plane curve $C$.

Suppose first that the curve $C$ is irreducible, of degree $N$. Denote by $a_{k}, k=1, \ldots, p$ the singular points of $C$, and let $r\left(C, a_{k}\right)$ be the number of irreducible branches of the germ $\left(C, a_{k}\right)$. Let $\nu: \tilde{C} \rightarrow C$ be the normalization mapping. Using the normalization map $\nu$ and the additivity of the Hodge-Deligne polynomial, it follows that,

$$
\begin{gathered}
P(C)=P\left(C \backslash(C)_{\text {sing }}\right)+P\left((C)_{\text {sing }}\right)=P\left(\tilde{C} \backslash\left(\cup_{k} \nu^{-1}\left(a_{k}\right)\right)+p=\right. \\
=P(\tilde{C})-\sum_{k} P\left(\nu^{-1}\left(a_{k}\right)\right)+p=u v-g u-g v+1-\sum_{k}\left(r\left(C, a_{k}\right)-1\right) .
\end{gathered}
$$

Indeed, it is known that for the smooth curve $\tilde{C}$, the genus $g=g(\tilde{C})$ is exactly the Hodge number $h^{1,0}(\tilde{C})=h^{0,1}(\tilde{C})$. Moreover, it is known that one has the formula

$$
g=\frac{(N-1)(N-2)}{2}-\sum_{k} \delta\left(C, a_{k}\right),
$$

relating the genus, the degree and the local singularities of $C$, and the $\delta$-invariants can be computed using the formula

$$
2 \delta\left(C, a_{k}\right)=\mu\left(C, a_{k}\right)+r\left(C, a_{k}\right)-1,
$$

where $\mu\left(C, a_{k}\right)$ is the Milnor number of the singularity $\left(C, a_{k}\right)$. For both formulas above, see Milnor, p. 85. This proves the following result.

Proposition 2.1. With the above notation and assumptions, we have the following for an irreducible plane curve $C \subset \mathbb{P}^{2}$.

(i) The Hodge-Deligne polynomial of $C$ is given by

$$
P(C)(u, v)=u v-g u-g v+1-\sum_{k}\left(r\left(C, a_{k}\right)-1\right),
$$

with $g$ given by the formula (2.1).

(ii) $H^{0}(C)=\mathbb{C}$ is pure of type $(0,0)$.

(iii) $H^{2}(C)=\mathbb{C}$ is pure of type $(1,1)$.

(iv) The mixed Hodge numbers of the MHS on $H^{1}(C)$ are given by

$$
h^{0,0}\left(H^{1}(C)\right)=\sum_{k}\left(r\left(C, a_{k}\right)-1\right), \quad h^{1,0}\left(H^{1}(C)\right)=h^{0,1}\left(H^{1}(C)\right)=g .
$$


In particular, one has the following formulas for the first Betti number of $C$.

$$
b_{1}(C)=\sum_{k}\left(r\left(C, a_{k}\right)-1\right)+2 g=(N-1)(N-2)-\sum_{k} \mu\left(C, a_{k}\right) .
$$

Now we consider the case of a curve $C$ having several irreducible components. More precisely, let $C=\bigcup_{j=1, r} C_{j}$ be the decomposition of $C$ as a union of irreducible components $C_{j}$, let $\nu_{j}: \tilde{C}_{j} \rightarrow C_{j}$ be the normalization mappings and set $g_{j}=g\left(\tilde{C}_{j}\right)$. Suppose that the curve $C_{j}$ has degree $N_{j}$, denote by $a_{k}^{j}$ for $k=1, \ldots, p_{j}$ be the singular points of $C_{j}$ and let $r\left(C_{j}, a_{k}^{j}\right)$ be the number of branches of the germ $\left(C_{j}, a_{k}^{j}\right)$. Then the formulas (2.1) and 2.2 can be applied to each irreducible curve $C_{j}$, as well as Proposition 2.1.

Let $A$ be the union of the singular sets of the curves $C_{j}$. Let $B$ be the set of points in $C$ sitting on at least two distinct components $C_{i}$ and $C_{j}$. For $b \in B$, let $n(b)$ be the number of irreducible components $C_{j}$ passing through $b$. By definition, $n(b) \geq 2$. Moreover, note that the sets $A$ and $B$ are not disjoint in general, and their union is precisely the singular set of $C$.

Using the additivity of Hodge-Deligne polynomials we get

$$
P(C)=P\left(C_{1} \cup \cdots \cup C_{r}\right)=\sum_{j=1}^{r} P\left(C_{j}\right)+(-1)^{l-1} \sum_{0 \leq i_{1}<\cdots<i_{l} \leq r} P\left(C_{i_{1}} \cap \cdots \cap C_{i_{l}}\right) .
$$

The first sum is easy to determine using Proposition 2.1.

$$
\sum_{j=1}^{r} P\left(C_{j}\right)=r u v-\left(\sum_{j=1}^{r} g_{j}\right) u-\left(\sum_{j=1}^{r} g_{j}\right) v+r-\sum_{j, k}\left(\left(r\left(C_{j}, a_{k}^{j}\right)-1\right) .\right.
$$

Consider now the alternated sum, where $l \geq 2$. The only points of $C$ that give a contribution to this sum are the points in $B$. Now, for a point $b \in B$, its contribution to the alternated sum is clearly given by

$$
c(b)=-\left(\begin{array}{c}
n(b) \\
2
\end{array}\right)+\left(\begin{array}{c}
n(b) \\
3
\end{array}\right)-\ldots+(-1)^{n(b)-1}\left(\begin{array}{c}
n(b) \\
n(b)
\end{array}\right)=-n(b)+1 .
$$

Proposition 2.2. With the above notation and assumptions, we have the following for a reducible plane curve $C=\bigcup_{j=1, r} C_{j}$.

(i) The Hodge-Deligne polynomial of $C$ is given by

$P(C)(u, v)=r u v-\left(\sum_{j=1}^{r} g_{j}\right) u-\left(\sum_{j=1}^{r} g_{j}\right) v+r-\sum_{j, k}\left(\left(r\left(C_{j}, a_{k}^{j}\right)-1\right)-\sum_{b \in B}(n(b)-1)\right.$.

with $g_{j}$ given by the formula (2.1).

(ii) $H^{0}(C)=\mathbb{C}$ is pure of type $(0,0)$.

(iii) $H^{2}(C)=\mathbb{C}^{r}$ is pure of type $(1,1)$.

(iv) The mixed Hodge numbers of the $M H S$ on $H^{1}(C)$ are given by

$$
h^{0,0}\left(H^{1}(C)\right)=\sum_{j, k}\left(\left(r\left(C_{j}, a_{k}^{j}\right)-1\right)+\sum_{b \in B}(n(b)-1)-r+1,\right.
$$




$$
h^{1,0}\left(H^{1}(C)\right)=h^{0,1}\left(H^{1}(C)\right)=\sum_{j} g_{j} .
$$

In particular, one has the following formula for the first Betti number of $C$.

$$
b_{1}(C)=\sum_{j, k}\left(\left(r\left(C_{j}, a_{k}^{j}\right)-1\right)+\sum_{b \in B}(n(b)-1)-r+1+2 \sum_{j} g_{j} .\right.
$$

Note that a point in the intersection $A \cap B$ will give a contribution to the last two sums in the above formula for $P(C)$.

Example 2.3. Suppose $C$ is a nodal curve. Then for each singularity $a_{k}^{j} \in A$ one has $a_{k}^{j} \notin B$ (otherwise we get worse singularities than nodes) and $r\left(a_{k}^{j}\right)=2$. Moreover, each point $b \in B$ satisfies $n(b)=2$. It follows that in this case we get

$$
P(C)(u, v)=r u v-\left(\sum_{j=1}^{r} g_{j}\right) u-\left(\sum_{j=1}^{r} g_{j}\right) v+r-n_{2},
$$

with $n_{2}$ the number of nodes of $C$. More precisely, in this case we have $n_{2}=n_{2}^{\prime}+n_{2}^{\prime \prime}$, where $n_{2}^{\prime}$ (resp. $n_{2}^{\prime \prime}$ ) is the number of nodes of $C$ in $A$ (resp. in $B$ ) and one clearly has

$$
n_{2}^{\prime}=S_{1}:=\sum_{j, k}\left(\left(r\left(C_{j}, a_{k}^{j}\right)-1\right), \quad n_{2}^{\prime \prime}=S_{2}:=\sum_{b \in B}(n(b)-1) .\right.
$$

Example 2.4. Suppose $C$ has only nodes and ordinary triple points as singularities. Then let $n_{3}$ be the number of triple points and note that we can write as above $n_{3}=$ $n_{3}^{\prime}+n_{3}^{\prime \prime}$, where $n_{3}^{\prime}$ (resp. $n_{3}^{\prime \prime}$ ) is the number of triple points of $C$ in $A_{0}=A \backslash B$ (resp. in $B$ ). For a point $a \in A_{0}$, the contribution to the sum $S_{1}$ is 2 , while the contribution to the sum $S_{2}$ is 0 .

A point $b \in B$ can be of two types. The first type, corresponding to the partition $3=1+1+1$, is when $b$ is the intersection of three components $C_{j}$, all smooth at $b$. The contribution of such a point $b$ is 0 to the sum $S_{1}$ and 2 to the sum $S_{2}$.

The second type, corresponding to the partition $3=2+1$, is when $b$ is the intersection of two components, say $C_{i}$ and $C_{j}$, such that $C_{i}$ has a node at $b$, and $C_{j}$ is smooth at $b$. The contribution of such a point $b$ is 1 to the sum $S_{1}$ and 1 to the sum $S_{2}$.

It follows that the contribution of any triple point to the sum $S_{1}+S_{2}$ is equal to 2 . Since the double points in $C$ can be treated exactly as in Example 2.3, this yields the following.

$$
P(C)(u, v)=r u v-\left(\sum_{j=1}^{r} g_{j}\right) u-\left(\sum_{j=1}^{r} g_{j}\right) v+r-n_{2}-2 n_{3} .
$$

When there are only triple points in $B$ of the first type, then we obviously have the following additional relations

$$
S_{1}=n_{2}^{\prime}+2 n_{3}^{\prime}, \quad S_{2}=n_{2}^{\prime \prime}+2 n_{3}^{\prime \prime} .
$$

Example 2.5. Suppose $C$ has only ordinary points of multiplicity 2,3 and 4 as singularities. Then let $n_{4}$ be the number of points of multiplicity 4 and note that we can write as above $n_{4}=n_{4}^{\prime}+n_{4}^{\prime \prime}$, where $n_{4}^{\prime}$ (resp. $\left.n_{4}^{\prime \prime}\right)$ is the number of points of multiplicity 
4 of $C$ in $A_{0}=A \backslash B$ (resp. in $B$ ). For a point $a \in A_{0}$ of multiplicity 4 , the contribution to the sum $S_{1}$ is 3 , while the contribution to the sum $S_{2}$ is 0 .

A point $b \in B$ can be of 4 types. The first type, corresponding to the partition $4=1+1+1+1$, is when $b$ is the intersection of 4 components $C_{j}$, all smooth at $b$. The contribution of such a point $b$ is 0 to the sum $S_{1}$ and 3 to the sum $S_{2}$.

The second type, corresponding to the partition $4=2+1+1$, is when $b$ is the intersection of 3 components, say $C_{i}, C_{j}$ and $C_{k}$, such that $C_{i}$ has a node at $b$, and $C_{j}$ and $C_{k}$ are smooth at $b$. The contribution of such a point $b$ is 1 to the sum $S_{1}$ and 2 to the sum $S_{2}$.

The third type, corresponding to the partition $4=2+2$, is when $b$ is the intersection of 2 components, say $C_{i}$ and $C_{k}$, such that $C_{i}$ and $C_{k}$ have a node at $b$. The contribution of such a point $b$ is 2 to the sum $S_{1}$ and 1 to the sum $S_{2}$.

The fourth type, corresponding to the partition $4=3+1$, is when $b$ is the intersection of 2 components, say $C_{i}$ and $C_{k}$, such that $C_{i}$ has a triple point at $b$, and $C_{k}$ is smooth at $b$. The contribution of such a point $b$ is 2 to the sum $S_{1}$ and 1 to the sum $S_{2}$.

It follows that the contribution of any point of multiplicity 4 to the sum $S_{1}+S_{2}$ is equal to 3. Since the double and triple points in $C$ can be treated exactly as in Example 2.4 , this yields the following.

$$
P(C)(u, v)=r u v-\left(\sum_{j=1}^{r} g_{j}\right) u-\left(\sum_{j=1}^{r} g_{j}\right) v+r-n_{2}-2 n_{3}-3 n_{4} .
$$

When there are only points of multiplicity 4 in $B$ of the first type, then we obviously have the following additional relations

$$
S_{1}=n_{2}^{\prime}+2 n_{3}^{\prime}+3 n_{4}^{\prime \prime}, \quad S_{2}=n_{2}^{\prime \prime}+2 n_{3}^{\prime \prime}+3 n_{4}^{\prime \prime} .
$$

Let's look now at the cohomology of the smooth surface $U=\mathbb{P}^{2} \backslash C$. By the additivity we get $P(U)=P\left(\mathbb{P}^{2}\right)-P(C)$ where $P\left(\mathbb{P}^{2}\right)=u^{2} v^{2}+u v+1$. This yields the following consequence.

\section{Corollary 2.6.}

$$
\begin{gathered}
P(U)(u, v)=u^{2} v^{2}-(r-1) u v+\left(\sum_{j=1}^{r} g_{j}\right) u+\left(\sum_{j=1}^{r} g_{j}\right) v-(r-1)+ \\
+\sum_{j, k}\left(\left(r\left(C_{j}, a_{k}^{j}\right)-1\right)+\sum_{b \in B}(n(b)-1) .\right.
\end{gathered}
$$

The contribution of $H_{c}^{4}(U, \mathbb{C})$ to $P(U)$ is the term $u^{2} v^{2}$, and that of $H_{c}^{3}(U, \mathbb{C})$ is the term $-(r-1) u v$. Moreover, the dimension $\operatorname{dim} G r_{F}^{1} H^{2}(U, \mathbb{C})$ is the number of independent classes of type $(1,2)$, which correspond to classes of type $(1,0)$ in $H_{c}^{2}(U)$, and hence to the terms in $u$ in $P(U)$. For both statements see the proof of Theorem 2.1 in [1]. This proves the following result.

\section{Theorem 2.7.}

$$
\operatorname{dim} G r_{F}^{1} H^{2}(U, \mathbb{C})=\sum_{j=1}^{r} g_{j}
$$


and

$$
\operatorname{dim} G r_{F}^{2} H^{2}(U, \mathbb{C})=\sum_{j=1}^{r} g_{j}+\sum_{j, k}\left(\left(r\left(C_{j}, a_{k}^{j}\right)-1\right)+\sum_{b \in B}(n(b)-1)-r+1 .\right.
$$

In particular, all the components $C_{j}$ of the curve $C$ are rational if and only if $H^{2}(U)$ is pure of type $(2,2)$.

Example 2.8. Suppose $C$ has only ordinary points of multiplicity 2, 3 and 4 as singularities. Then let $n_{k}$ be the number of points of multiplicity $k$, for $k=2,3,4$ Then using Example 2.5, we get the formula

$$
\operatorname{dim} G r_{F}^{2} H^{2}(U, \mathbb{C})=\sum_{j=1}^{r} g_{j}-r+1+n_{2}+2 n_{3}+3 n_{4}
$$

\section{Arrangements of transversely intersecting CURVES}

Recall that $C=\bigcup_{j=1, r} C_{j}$ is the decomposition of $C$ as a union of irreducible components $C_{j}$, and the curve $C_{j}$ has degree $N_{j}$. In this section we assume that any curve $C_{j}$ has only ordinary multiple points as singularities and let $n_{k}\left(C_{j}\right)$ denote the number of ordinary points on $C_{j}$ of multiplicity $k$. We also assume that the intersection of any two distinct components $C_{i}$ and $C_{j}$ is transverse, i.e. the points in $C_{i} \cap C_{j}$ are nodes of the curve $C_{i} \cup C_{j}$. This implies in particular that $A \cap B=\emptyset$. The formulas (2.1) and (2.2) yield the equality.

$$
g_{j}=\frac{\left(N_{j}-1\right)\left(N_{j}-2\right)}{2}-\frac{1}{2} \sum_{k}\left(\mu\left(C_{j}, a_{k}^{j}\right)+r\left(C, a_{k}^{j}\right)-1\right),
$$

Using this, Theorem 2.7 gives the formula

$$
\begin{gathered}
\operatorname{dim} G r_{F}^{2} H^{2}(U, \mathbb{C})=\sum_{j=1}^{r} \frac{\left(N_{j}-1\right)\left(N_{j}-2\right)}{2}-\frac{1}{2} \sum_{j, k}\left(\mu\left(C_{j}, a_{k}^{j}\right)-r\left(C, a_{k}^{j}\right)+1\right)+ \\
+\sum_{b \in B}(n(b)-1)-r+1 .
\end{gathered}
$$

If $a_{k}^{j}$ is an ordinary $m$-multiple point on the curve $C_{j}$, one has $\mu\left(C_{j}, a_{k}^{j}\right)=(m-1)^{2}$ and hence

$$
\mu\left(C_{j}, a_{k}^{j}\right)-r\left(C, a_{k}^{j}\right)+1=(m-1)(m-2) .
$$

If we denote by $n_{m}^{\prime}$ (resp. $n_{m}^{\prime \prime}$ ) the number of $m$-multiple points of $C$ coming from just one component $C_{j}$ (resp. from the intersection of several components $C_{j}$ ), we see that we have

$$
\sum_{j, k}\left(\mu\left(C_{j}, a_{k}^{j}\right)-r\left(C, a_{k}^{j}\right)+1\right)=\sum_{m}(m-1)(m-2) n_{m}^{\prime} .
$$

This equality explains the contribution of the points in $A$. Now let $b \in B$ such that $n(b)=m$. The number of such points is precisely $n_{m}^{\prime \prime}$. It follows that

$$
\sum_{b \in B}(n(b)-1)=\sum_{m}(m-1) n_{m}^{\prime \prime} .
$$


Let $1 \leq i<j \leq r$ and consider the intersection $C_{i} \cap C_{j}$. It contains exactly $N_{i} N_{j}$ points, since $C_{i}$ and $C_{j}$ intersects transversely. The sum $S=\sum_{1 \leq i<j \leq r} N_{i} N_{j}$ represents the number of all such intersection points. Note that a point $b \in B$ is counted in this sum exactly $\left(\begin{array}{c}n(b) \\ 2\end{array}\right)$ times. This yields the following formula

$$
2 S=\sum_{m} m(m-1) n_{m}^{\prime \prime}
$$

These formulas give the following result.

Theorem 3.1. With the above assumptions and notation, one has

$$
\operatorname{dim} G r_{F}^{2} H^{2}(U, \mathbb{C})=\frac{(N-1)(N-2)}{2}-\sum_{m}\left(\begin{array}{c}
m-1 \\
2
\end{array}\right) n_{m}
$$

with $n_{m}=n_{m}^{\prime}+n_{m}^{\prime \prime}$ the number of ordinary $m$-tuple points of $C$.

The following consequence of Theorem 2.7 and Theorem 3.1 applies in particular to any projective line arrangement.

Corollary 3.2. Assume that $C=\bigcup_{j=1, r} C_{j}$ is the decomposition of $C$ as a union of irreducible components $C_{j}$, with any curve $C_{j}$ having only ordinary multiple points as singularities and being rational, i.e. $g_{j}=0$. If the intersection of any two distinct components $C_{i}$ and $C_{j}$ is transverse, i.e. the points in $C_{i} \cap C_{j}$ are nodes of the curve $C_{i} \cup C_{j}$, then one has

$$
\operatorname{dim} H^{2}(U, \mathbb{C})=\frac{(N-1)(N-2)}{2}-\sum_{m}\left(\begin{array}{c}
m-1 \\
2
\end{array}\right) n_{m},
$$

with $n_{m}$ the number of ordinary m-tuple points of $C$.

\section{Curves with ordinary singularities of multiplicity $\leq 4$}

Let $C \subset \mathbb{P}^{2}$ be a curve of degree $N$ having only ordinary singular points of multiplicity at most 4 . Set $U=\mathbb{P}^{2} \backslash C$, and let $C=\cup_{j=1}^{r} C_{j}$ be the decomposition of $C$ in irreducible components. Then,

$$
\begin{aligned}
P(C) & =\sum_{j=1}^{r} P\left(C_{j}\right)-\sum_{0 \leq i<j \leq r} P\left(C_{i} \cap C_{j}\right)+\sum_{0 \leq i<j<k \leq r} P\left(C_{i} \cap C_{j} \cap C_{k}\right) \\
& -\sum_{0 \leq i<j<k<l \leq r} P\left(C_{i} \cap C_{j} \cap C_{k} \cap C_{l}\right) .
\end{aligned}
$$

Let $a_{m}^{j}$ denote the number of singular points of multiplicity $m$ that belong to the component $C_{j}$ (note that a point can be singular on two components, being a node on each of them).

Denote by $b_{3}^{k}$ (respectively $b_{4}^{k}$ ) the number of triple points (respectively points of multiplicity 4) of $C$ that are intersection of exactly $k$ components, for $k=2,3$ (respectively $k=3,4$ ). Let $b_{4}^{2}$ (respectively $\tilde{b}_{4}^{2}$ ) be the number of singular points $p$ of multiplicity 4 
in $C$ representing the intersection of exactly 2 components, such that one of which has a triple point at $p$ (respectively each one has a node at $p$ ). Then one has

$$
\sum_{0 \leq i<j \leq r} P\left(C_{i} \cap C_{j}\right)=\sum_{0 \leq i<j \leq r} N_{i} N_{j}-b_{3}^{2}-3 \tilde{b_{4}^{2}}-2 b_{4}^{2}-2 b_{4}^{3} .
$$

Indeed, a point of type $b_{3}^{2}$ (resp. $b_{4}^{2}$, resp. $\left.\tilde{b}_{4}^{2}\right)$ occurs only in one intersection $C_{i} \cap C_{j}$, and has the multiplicy 2 (resp.3, resp. 4) in this intersection. A point of type $b_{4}^{3}$ occurs in 3 intersections $C_{i} \cap C_{j}$ with multiplicitities $1,2,2$, and this accounts for the correction term $-2 b_{4}^{3}$. Then one has

$$
\sum_{0 \leq i<j<k \leq r} P\left(C_{i} \cap C_{j} \cap C_{k}\right)=b_{3}^{3}+b_{4}^{3}+\left(\begin{array}{l}
4 \\
3
\end{array}\right) b_{4}^{4},
$$

and

$$
\sum_{0 \leq i<j<k<l \leq r} P\left(C_{i} \cap C_{j} \cap C_{k} \cap C_{l}\right)=b_{4}^{4} .
$$

Hence, by Proposition 2.1, we get the following.

$$
\begin{aligned}
P(C) & =r u v-\left(\sum_{j=1}^{r} g_{j}\right) u-\left(\sum_{j=1}^{r} g_{j}\right) v-\sum_{j=1}^{r}\left(a_{2}^{j}+2 a_{3}^{j}+3 a_{4}^{j}\right)-\sum N_{i} N_{j} \\
& +b_{3}^{2}+3 \tilde{b_{4}^{2}}+2 b_{4}^{2}+3 b_{4}^{3}+b_{3}^{3}+3 b_{4}^{4} .
\end{aligned}
$$

Therefore, as above, we obtain

$$
\begin{aligned}
P(U) & =u^{2} v^{2}-(r-1) u v+1-r+\left(\sum_{j=1}^{r} g_{j}\right) u+\left(\sum_{j=1}^{r} g_{j}\right) v+\sum_{j=1}^{r}\left(a_{2}^{j}+3 a_{3}^{j}+6 a_{4}^{j}\right) \\
& -\sum_{j=1}^{r}\left(a_{3}^{j}+3 a_{4}^{j}\right)+\sum N_{i} N_{j}-b_{3}^{2}-3 \tilde{b}_{4}^{2}-2 b_{4}^{2}-3 b_{4}^{3}-b_{3}^{3}-3 b_{4}^{4} .
\end{aligned}
$$

Finally we get

$$
\begin{aligned}
\operatorname{dim} G r_{F}^{2} H^{2}(U) & =\sum_{j=1}^{r}\left(g_{j}+a_{2}^{j}+3 a_{3}^{j}+6 a_{4}^{j}-1\right)+\sum N_{i} N_{j}+1-\left(\sum_{j=1}^{r} a_{3}^{j}+b_{3}^{2}+b_{3}^{3}\right) \\
& -3\left(\sum_{j=1}^{r} a_{4}^{j}+\tilde{b}_{4}^{2}+b_{4}^{2}+b_{4}^{3}+b_{4}^{4}\right)+b_{4}^{2} \\
& =\frac{(N-1)(N-2)}{2}-n_{3}-3 n_{4}+b_{4}^{2}
\end{aligned}
$$

with $n_{m}$ the number of ordinary $m$-tuple points of $C$.

Theorem 4.1. Let $C \subset \mathbb{P}^{2}$ be a curve of degree $N$ having only ordinary singular points of multiplicity at most 4. If $U=\mathbb{P}^{2} \backslash C$, then one has

$$
\operatorname{dim} G r_{F}^{2} H^{2}(U, \mathbb{C})=\frac{(N-1)(N-2)}{2}-\sum_{m=3,4}\left(\begin{array}{c}
m-1 \\
2
\end{array}\right) n_{m}+b_{4}^{2},
$$


with $n_{m}$ the number of ordinary $m$-tuple points of $C$ and $b_{4}^{2}$ the number of singular points $p$ of $C$ which are smooth on one component $C_{i}$ of $C$ and have multiplicity 3 on the other component $C_{j}$ of $C$ passing through $p$.

\section{Pole order filtration versus Hodge filtration for Plane Curve COMPLEMENTS}

For any hypersurface $V$ in a projective space $\mathbb{P}^{n}$, the cohomology groups $H^{*}(U, \mathbb{C})$ of the complement $U=\mathbb{P}^{n} \backslash V$ have a pole order filtration $P^{k}$, see for instance [8], and it is known by the work of P. Deligne, A. Dimca [3] and M. Saito [11] that one has

$$
F^{k} H^{m}(U, \mathbb{C}) \subset P^{k} H^{m}(U, \mathbb{C})
$$

for any $k$ and any $m$. For $m=0$ and $m=1$, the above inclusions are in fact equalities (the case $m=0$ is obvious and the case $m=1$ follows from the equality $F^{1} H^{1}(U, \mathbb{C})=$ $\left.H^{1}(U, \mathbb{C})\right)$. For $m=2$, we have again $F^{k} H^{2}(U, \mathbb{C})=P^{k} H^{2}(U, \mathbb{C})$ for $k=0,1$ for obvious reasons, but one may get strict inclusions

$$
F^{2} H^{2}(U, \mathbb{C}) \neq P^{2} H^{2}(U, \mathbb{C})
$$

already in the case when $V=C$ is a plane curve, see [5], Remark 2.5 or [4]. However, to give such examples of plane curves was until now rather complicated. We give below a numerical condition which tells us exactly when the above strict inclusion holds.

We need first to recall some basic definitions. Let $S=\oplus_{r} S_{r}=\mathbb{C}[x, y, z]$ be the graded ring of polynomials with complex coefficients, where $S_{r}$ is the vector space of homogeneous polynomials of $S$ of degree $r$. For a homogeneous polynomial $f$ of degree $N$, define the Jacobian ideal of $f$ to be the ideal $J_{f}$ generated in $S$ by the partial derivatives $f_{x}, f_{y}, f_{z}$ of $f$ with respect to $x, y$ and $z$. The graded Milnor algebra of $f$ is given by

$$
M(f)=\oplus_{r} M(f)_{r}=S / J_{f} .
$$

Note that the dimensions $\operatorname{dim} M(f)_{r}$ can be easily computed in a given situation using some computer software e.g. Singular. Now we can state the main result of this section.

Theorem 5.1. Let $C: f=0$ be a reduced curve of degree $N$ in $\mathbb{P}^{2}$ having only weighted homogeneous singularities and let $C_{i}$ for $i=1, \ldots, r$ be the irreducible components of $C$. If $U=\mathbb{P}^{2} \backslash C$, then

$$
\operatorname{dim} P^{2} H^{2}(U, \mathbb{C})-\operatorname{dim} F^{2} H^{2}(U, \mathbb{C})=\tau(C)+\sum_{i=1, r} g_{i}-\operatorname{dim} M(f)_{2 N-3},
$$

where $\tau(C)$ is the global Tjurina number of $C$ (that is the sum of the Tjurina numbers of all the singularities of $C$ ) and $g_{i}$ is the genus of the normalization of $C_{i}$ for $i=1, \ldots, r$.

In particular we get the following result, which yields in particular a new proof for Theorem 1.3 in [7].

Corollary 5.2. If a reduced plane curve has only nodes as singularities, then one has

$$
\operatorname{dim} M(f)_{2 N-3}=\tau(C)+\sum_{i=1, r} g_{i}
$$


Proof. Indeed, it is known that for a nodal curve one has the equality $F^{2} H^{2}(U, \mathbb{C})=$ $P^{2} H^{2}(U, \mathbb{C})$, see [2] or [11].

Note that we have the following obvious consequence of Theorem 2.7 .

Corollary 5.3. For a reduced plane curve $C$ one has

$$
\operatorname{dim} P^{2} H^{2}(U, \mathbb{C})-\operatorname{dim} F^{2} H^{2}(U, \mathbb{C}) \leq \sum_{i=1, r} g_{i}
$$

Proof. Indeed, Theorem 2.7 can be restated as

$$
\operatorname{dim} H^{2}(U, \mathbb{C})-\operatorname{dim} F^{2} H^{2}(U, \mathbb{C})=\sum_{i=1, r} g_{i},
$$

in view of the equality $F^{1} H^{2}(U, \mathbb{C})=H^{2}(U, \mathbb{C})$, see [4], proof of Corollary 1.32, page 185 .

Remark 5.4. If a reduced plane curve $C$ has only rational irreducible components, i.e. $g_{i}=0$ for all $i$, then the above inequality implies $F^{2} H^{2}(U, \mathbb{C})=P^{2} H^{2}(U, \mathbb{C})$. This result can be regarded as an improvement of a part of the Remark 2.5 in [5], where the result is claimed only for curves with nodes and cusps as singularities.

The above discussion implies also the following result, which can be regarded as a generalization of Theorem 4.1 (A) in [1].

Corollary 5.5. If a reduced plane curve $C: f=0$ has only weighted homogeneous singularities, then one has

$$
0 \leq \operatorname{dim} M(f)_{2 N-3}-\tau(C) \leq \sum_{i=1, r} g_{i} .
$$

In particular, if in addition the curve $C$ has only rational irreducible components, then one has

$$
\operatorname{dim} M(f)_{2 N-3}=\tau(C) .
$$

Now we give the proof of Theorem 5.1. Corollary 1.3 in [8] implies that

$$
\operatorname{dim} P^{2} H^{2}(U, \mathbb{C})=\operatorname{dim} H^{2}(U, \mathbb{C})+\tau(C)-\operatorname{dim} M(f)_{2 N-3} .
$$

On the other hand, Theorem 2.7 and the fact $\operatorname{dim} F^{1} H^{2}(U, \mathbb{C})=H^{2}(U, \mathbb{C})$ yield

$$
\operatorname{dim} F^{2} H^{2}(U, \mathbb{C})=\operatorname{dim} H^{2}(U, \mathbb{C})-\sum_{i=1, r} g_{i},
$$

which clearly completes the proof of Theorem 5.1.

Example 5.6. In this example we present a free divisor $C: f=0$, whose irreducible components consist of 12 lines and one elliptic curve, and where $F^{2} H^{2}(U, \mathbb{C}) \neq$ $P^{2} H^{2}(U, \mathbb{C})$. Let $f=x y z\left(x^{3}+y^{3}+z^{3}\right)\left[\left(x^{3}+y^{3}+z^{3}\right)^{3}-27 x^{3} y^{3} z^{3}\right]$. If we consider the pencil of cubic curves $\left(x^{3}+y^{3}+z^{3}, x y z\right)$, then the curve $C$ contains all the singular fibers of this pencil, and this accounts for the 12 lines given by

$$
x y z\left[\left(x^{3}+y^{3}+z^{3}\right)^{3}-27 x^{3} y^{3} z^{3}\right]=0,
$$


and the elliptic curve (hence of genus 1) given by $x^{3}+y^{3}+z^{3}=0$. Then $C$ is a free divisor, see [13] or by a direct computation using Singular, which shows that $I=J_{f}$, where $I$ is the saturation of the Jacobian ideal $J_{f}$, see Remark 4.7 in [6]. The direct computation by Singular also yields $\tau(C)=156$ and $\operatorname{dim} M(f)_{2 N-3}=\operatorname{dim} M(f)_{27}=156$. Moreover, applying Corollary 1.5 in [9], we see via a Singular computation that all singularities of the curve $C$ are weighted homogeneous. Alternatively, there are 12 nodes, 3 in each of the 4 singular fibers of the pencils (which are triangles), and the 9 base points of the pencil, each an ordinary point of multiplicity 5. Each of the 12 lines contains exactly 3 of these base points, and they are exactly the intersection of the elliptic curve with the line. This description implies that there are no other singularities, in accord with

$$
12+9 \times 16=156=\tau(C) .
$$

It follows from Theorem 5.1 that $\operatorname{dim} P^{2} H^{2}(U, \mathbb{C})-\operatorname{dim} F^{2} H^{2}(U, \mathbb{C})=1$. Hence the presence of a single irrational component of $C$ leads to $F^{2} H^{2}(U, \mathbb{C}) \neq P^{2} H^{2}(U, \mathbb{C})$.

Acknowledegment: I gratefully acknowledge the support of the Lebanese National Council for Scientific Research, without which the present study could not have been completed.

\section{REFERENCES}

[1] N. Abdallah: On plane curves with double and triple points, arXiv:1401.6032, to appear in Mathematica Scandinavica.

[2] P. Deligne: Théorie de Hodge II, Publ. Math. IHES, 40 (1971), 5-58.

[3] P. Deligne, A. Dimca: Filtrations de Hodge et par l'ordre du pôle pour les hypersurfaces singulières, Ann. Sci. Ecole Norm. Sup. (4) 23(1990), 645-656.

[4] A. Dimca: Singularities and Topology Hypersurface (Universitext, Springer-Verlag, 1992).

[5] A. Dimca, M. Saito: A Generalization on Griffiths' Theorem on Rational Integrals, Duke Math. J. 135(2006),303-326.

[6] A. Dimca, E. Sernesi: Syzygies and logarithmic vector fields along plane curves, Journal de l'École polytechnique-Mathématiques 1(2014), 247-267.

[7] A. Dimca, G. Sticlaru: Chebyshev Curves, Free Resolutions and Rational Curve Arrangements, Math. Proc. Camb. Phil. Soc. 1-13, 2012

[8] A. Dimca, G. Sticlaru: Koszul complexes and pole order filtrations, Proc. Edinburgh Math. Soc. doi:10.1017/S0013091514000182.

[9] A. Dimca, G. Sticlaru: Hessian ideals of a homogeneous polynomial and generalized Tjurina algebras, arXiv:1412.0632.

[10] Ph. Griffiths: On the Period of certain Rational Integrals I, II, Ann. Math. 90(1969), 460-541.

[11] M. Saito: On b-function, spectrum and rational singularity, Math. Ann. 295(1993), 51-74.

[12] E. Sernesi: The local cohomology of the jacobian ring, Documenta Mathematica, 19 (2014), 541565.

[13] J. Vallès: Free divisors in a pencil of curves, arXiv:1502.02416

[14] C. Voisin: Théorie de Hodge et Géométrie algébrique complexe, Société mathématique de France 2002.

Univ. Nice Sophia Antipolis, CNRS, LJAD, UMR 7351, 06100 Nice, France.

E-mail address: Nancy.ABDALLAH@unice.fr 